\title{
NONLINEAR MONOTONE OPERATORS AND CONVEX SETS IN BANACH SPACES
}

\author{
BY FELIX E. BROWDER ${ }^{1}$ \\ Communicated May 10, 1965
}

Introduction. Let $X$ be a real Banach space, $X^{*}$ its conjugate space, $(w, u)$ the pairing between $w$ in $X^{*}$ and $u$ in $X$. If $C$ is a closed convex subset of $X$, a mapping $T$ of $C$ into $X^{*}$ is said to be monotone if

$$
(T u-T v, u-v) \geqq 0
$$

for all $u$ and $v$ in $C$.

It is the object of the present note to prove the following theorem:

TheOREM 1. Let $C$ be a closed convex subset of the reflexive Banach space $X$ with $0 \in C, T$ a monotone mapping of $C$ into $X^{*}$. Suppose that $T$ is continuous from line segments in $C$ to the weak topology of $X^{*}$ while $(T u, u) /\|u\| \rightarrow+\infty$ as $\|u\| \rightarrow+\infty$.

Then for each given element $w_{0}$ of $X^{*}$, there exists $u_{0}$ in $C$ such that

$$
\left(T u_{0}-w_{0}, u_{0}-v\right) \leqq 0
$$

for all $v$ in $C$.

If $C=X$, Theorem 1 asserts that $T u_{0}=w_{0}$ and reduces to a theorem on monotone operators proved independently by the writer [1] and G. J. Minty [9] and applied to nonlinear elliptic boundary value problems by the writer in [2], [3], and [6]. (See also Leray and Lions [7].) If $C=V$, a closed subspace of $X$, the conclusion of Theorem 1 is that $T u_{0}-w_{0} \in V^{\perp}$, which yields a variant of the generalized form of the Beurling-Livingston theorem proved by the writer in [4] and [5]. The conclusion of Theorem 1 for $C=X$ was extended by the writer to classes of densely defined operators (see [6] for references) and in [5] to multivalued mappings.

It is easily shown that Theorem 1 generalizes and includes as a special case the following linear theorem of Stampacchia, which has been applied by the latter to the proof of the existence of capacitary potentials with respect to second-order linear elliptic equations with discontinuous coefficients: ${ }^{2}$

TheORem 2. Let $H$ be a real Hilbert space, $C$ a closed convex subset of $H, a(u, v)$ a bilinear form on $H$ which is separately continuous in $u$

1 The preparation of this paper was partially supported by NSF Grant GP 3552 .

2 C. R. Acad. Sci. Paris 258 (1964), 4413-4416.

Added in proof. A result similar to Theorem 1 has recently been obtained jointly by Hartman and Stampacchia (in an as yet unpublished paper) who also give a very interesting application to existence theorems for second order nonlinear elliptic equations. 
and $v$. Suppose that there exists a constant $c>0$ such that $a(u, u)$ $\geqq c\|u\|^{2}$ for all $u$ in $H$.

Then for each $w_{0}$ in $H$, there exists $u_{0}$ in $C$ such that

$$
a\left(u_{0}, u_{0}-v\right) \leqq\left(w_{0}, u-v\right)
$$

for all $v$ in $C$.

1. We denote weak convergence by $\rightarrow$, strong convergence by $\rightarrow$.

Lemмa 1. If $u_{0} \in C, u_{0}$ is a solution of the inequality (2) if and only if

$$
\left(T v-w_{0}, v-u_{0}\right) \geqq 0
$$

for all $v$ in $C$.

Proof of Lemma 1. If for a given $u_{0}$ in $C$ and all $v$ in $C$, we have $\left(T u_{0}-w_{0}, u_{0}-v\right) \leqq 0$, then since

$$
\left(T u_{0}-T v, u_{0}-v\right) \geqq 0
$$

by monotonicity, it follows that

$$
\left(T v, u_{0}-v\right) \leqq\left(T u_{0}, u_{0}-v\right) \leqq\left(w_{0}, u_{0}-v\right),
$$

i.e.,

$$
\left(T v-w_{0}, v-u_{0}\right) \leqq 0 .
$$

Conversely, suppose the inequality (4) holds for all $v$ in $C$. Suppose $v_{0} \in C$, and for $0<t \leqq 1$, set

$$
v_{t}=(1-t) u_{0}+t v_{0} .
$$

Then $v_{t} \in C, v_{t}-u_{0}=t\left(v_{0}-u_{0}\right)$, and we have

$$
0 \leqq\left(T v_{t}-w_{0}, t\left(v_{0}-u_{0}\right)\right)=t\left(T v_{t}-w_{0}, v_{0}-u_{0}\right)
$$

Since $t>0$ may be canceled, we have

$$
\left(T v_{t}-w_{0}, v_{0}-u_{0}\right) \geqq 0 \text {. }
$$

If we let $t \rightarrow 0$ and use the weak continuity of $T$ on segments in $C$, we have $T v_{t} \rightarrow T u_{0}$, and hence

$$
\left(T u_{0}-w_{0}, u_{0}-v_{0}\right) \leqq 0 . \text { q.e.d. }
$$

Definition. Let $c(r)=\inf _{\|u\|=r}\{(T u, u) /\|u\|\}$. By the hypothesis of Theorem $1, c(r) \rightarrow+\infty$ as $r \rightarrow+\infty$. We have

$$
(T u, u) \geqq c(\|u\|)\|u\|, \quad u \in C .
$$

LEMmA 2. There exists a constant $M$ which depends only upon the 
function $c(r)$ and on $\left\|w_{0}\right\|$ such that if $u_{0}$ is a solution of the inequality (2), then $\left\|u_{0}\right\| \leqq M$.

Proof of Lemma 2. If

$$
\left(T u_{0}-w_{0}, u_{0}-v\right) \leqq 0, \quad v \in C,
$$

we have since $0 \in C$,

$$
c\left(\left\|u_{0}\right\|\right)\left\|u_{0}\right\| \leqq\left(T u_{0}, u_{0}\right) \leqq\left(T u_{0}-w_{0}, u_{0}\right)+\left(w_{0}, u_{0}\right) \leqq\left\|w_{0}\right\| \cdot\left\|u_{0}\right\| .
$$

Hence

$$
c\left(\left\|u_{0}\right\|\right) \leqq\left\|w_{0}\right\|
$$

and

$$
\left\|u_{0}\right\| \leqq M\left(\left\|w_{0}\right\|, c(r)\right) \text {. q.e.d. }
$$

Definition. If $G \subset X \times X^{*}, G$ is said to be a monotone set if $[u, w]$, $\left[u_{1}, w_{1}\right] \in G$ implies that $\left(w-w_{1}, u-u_{1}\right) \geqq 0$.

$G$ is said to be maximal monotone if it is monotone and maximal in the monotone sets ordered by inclusion.

Lemma 3. Under the hypotheses of Theorem 1, suppose that $C$ has 0 as an interior point and let $G \subset X \times X^{*}$ be given by $G=\{[u, w] \mid u \in C, w=T u+z$, where $(z, u-v) \geqq 0$ for all $v$ in $C\}$.

Then $G$ is a maximal monotone set in $X \times X^{*}$.

Proof of Lemma 3. $G$ is a monotone set since if $[u, w]$ and $\left[u_{1}, w_{1}\right]$ $\in G$, with $w=T u+z, w_{1}=T u_{1}+z_{1}$, then

$$
\begin{aligned}
& \left(w-w_{1}, u-u_{1}\right) \\
& \quad=\left(T u-T u_{1}, u-u_{1}\right)+\left(z, u-u_{1}\right)+\left(z_{1}, u_{1}-u\right) \geqq 0 .
\end{aligned}
$$

Suppose on the other hand that $\left[u_{0}, w_{0}\right] \in X \times X^{*}$ with

$$
\left(w_{0}-w, u_{0}-u\right) \geqq 0
$$

for all $[u, w]$ in $G$. We assert first that $u_{0} \in C$. Otherwise, $u_{0}=s v_{0}$ for some $v_{0}$ on the boundary of $C$ with $s>1$. Let $z_{0}=0$ be an element of $X^{*}$ such that $\left(z_{0}, v_{0}-v\right) \geqq 0$ for all $v$ in $C$. Since 0 is an interior point of $C,\left(z_{0}, v_{0}\right)>0$. For each $\lambda>0,\left[v_{0}, T v_{0}+\lambda z_{0}\right]$ lies in $G$. Hence

$$
0 \leqq\left(w_{0}-T v_{0}-\lambda z_{0}, u_{0}-v_{0}\right)=(s-1)\left(w_{0}-T v_{0}-\lambda z_{0}, v_{0}\right) .
$$

Cancelling $(s-1)>0$, we have

$$
\lambda\left(z_{0}, v_{0}\right) \leqq\left(w_{0}, v_{0}\right)-\left(T v_{0}, v_{0}\right),
$$


which is a contradiction since $\left(z_{0}, v_{0}\right)>0$ and $\lambda$ is arbitrary. Hence $u_{0} \in C$.

In addition, for each $u$ in $C,[u, T u]$ lies in $G$. Hence

$$
\left(T u-w_{0}, u-u_{0}\right) \geqq 0 .
$$

Applying Lemma 1, we have

$$
\left(T u_{0}-w_{0}, u_{0}-v\right) \leqq 0, \quad v \in C .
$$

Hence $T u_{0}-w_{0}=-z$, where $\left(z, u_{0}-v\right) \geqq 0$ for all $v$ in $C$. Hence $w_{0}=T u_{0}+z$, and $\left[u_{0}, w_{0}\right] \in G$. q.e.d.

LEMMA 4. Theorem 1 holds if $X$ is a finite dimensional Banach space $F$.

Proof of Lemma 4. We may suppose without loss of generality that $w_{0}=0$, that $F$ is a finite dimensional Hilbert space with $F^{*}=F$, and that $C$ spans $F$ and hence has an interior point $v_{0}$ in $F$. Replacing $C$ by $C_{0}=v_{0}-C$ and defining a new mapping $T^{\prime}$ on $C_{0}$ by $T^{\prime} u=-T\left(v_{0}-u\right)$, it is easy to verify that we may assume that 0 is an interior point of $C$ and the condition on $(T u, u)$ is replaced by

$$
\left(T u, u-v_{0}\right) \geqq c(\|u\|)\|u\|
$$

for a given $v_{0}$ in $C$, with $c(r) \rightarrow+\infty$ as $r \rightarrow+\infty$.

Let $G$ be the maximal monotone set in $F \times F^{*}$ constructed in Lemma 3. Then $n G$ is maximal monotone for each positive integer $n$. By a theorem of Minty [8], for each $n>0$, there exists $\left[u_{n}, w_{n}\right] \in G$ such that

$$
u_{n}+n w_{n}=0 .
$$

Since $w_{n}=T u_{n}+z_{n}$, where $\left(z_{n}, u_{n}-v\right) \geqq 0$ for all $v$ in $C$, we have

$$
\begin{aligned}
-\left(\frac{1}{n} u_{n}, u_{n}-v_{0}\right) & =\left(w_{n}, u_{n}-v_{0}\right) \\
& =\left(T u_{n}, u_{n}-v_{0}\right)+\left(z_{n}, u_{n}-v_{0}\right) \geqq c\left(\left\|u_{n}\right\|\right)\left\|u_{n}\right\|,
\end{aligned}
$$

while

$$
-\left(\frac{1}{n} u_{n}, u_{n}-v_{0}\right) \leqq \frac{1}{n}\left\|u_{n}\right\| \cdot\left\|v_{0}\right\| .
$$

Thus $c\left(\left\|u_{n}\right\|\right) \leqq n^{-1}\left\|v_{0}\right\|$, and $\left\|u_{n}\right\| \leqq M$, independent of $n$.

We may extract a subsequence which we again denote by $u_{n}$ such that $u_{n} \rightarrow u_{0}$ in $F$. Then $w_{n} \rightarrow 0$. For each $u$ in $C$

$$
\left(T u-w_{n}, u=u_{n}\right) \geqq 0 .
$$


Taking the limit as $n \rightarrow \infty$, we have

$$
\left(T u, u-u_{0}\right) \geqq 0, \quad u \in C .
$$

By Lemma 1,

$$
\left(T u_{0}, u_{0}-v\right) \leqq 0
$$

for all $v$ in $C$. q.e.d.

Proof of Theorem 1. It suffices to take $w_{0}=0$. For each finite dimensional subspace $F$ of $X$, let $C_{F}=C \cap F, j_{F}$ be the injection map of $F$ into $X, j_{F}^{*}$ the dual projection map of $X^{*}$ onto $F^{*}$. We set

$$
T_{F}=j_{F}^{*}\left(T \mid C_{F}\right): C_{F} \rightarrow F^{*} .
$$

Then $T_{F}$ satisfies the hypotheses of Lemma 4 , and there exists $u_{F}$ in $C_{F}$ such that

$$
\left(T_{F} u_{F}, u_{F}-v\right)=\left(T u_{F}, u_{F}-v\right) \leqq 0, \quad v \in C_{F} .
$$

By Lemma 2, since for $u$ in $C_{F}$,

$$
\left(T_{F} u, u\right)=(T u, u) \geqq c(\|u\|)\|u\|,
$$

there exists a constant $M$ independent of $F$ such that $\left\|u_{F}\right\| \leqq M$. Since $X$ is reflexive and $C$ is weakly closed, there exists $u_{0}$ in $C$ such that for every finite dimensional $F, u_{0}$ lies in the weak closure of the set $V_{F}=U_{F \subset P_{1}}\left\{u_{F_{1}}\right\}$.

Let $v$ be an arbitrary element of $C, F$ a finite dimensional subspace of $X$ which contains $v$. For $u_{F_{1}}$ in $V_{F}$, by Lemma 1 ,

$$
\left(T v, v-u_{F_{1}}\right) \geqq 0 \text {. }
$$

Since $\left(T v, v-v_{1}\right)$ is weakly continuous in $v_{1}$, we have

$$
\left(T v, v-u_{0}\right) \leqq 0, \quad v \in C .
$$

By Lemma 1, $\left(T u_{0}, u_{0}-v\right) \geqq 0$ for $v$ in $C$. q.e.d.

\section{BIBLIOGRAPHY}

1. F. E. Browder, Nonlinear elliptic boundary value problems, Bull. Amer. Math. Soc. 69 (1963), 862-874.

2. - Nonlinear elliptic problems. II, Bull. Amer. Math. Soc. 70 (1964), 299-301.

3. - Nonlinear elliptic boundary value problems. II, Trans. Amer. Math. Soc. 117 (1965), 530-550.

4. - On a theorem of Beurling and Livingston, Canad. J. Math. 17 (1965), $367-372$.

5. - Multivalued monotone nonlinear mappings and duality mappings in Banach spaces, Trans. Amer. Math. Soc. 118 (1965) 338-351. 
6. - Existence and uniqueness theorems for solutions of nonlinear boundary value problems, Proc. Sympos. Appl. Math., Vol. 17, Amer. Math. Soc., Providence, R. I., 1965; pp. 24-29.

7. J. Leray and J. L. Lions, Quelques résultats de Visik sur les problèmes elliptiques quasi-linéaires par le methode de Minty-Browder, Séminaire de Collège de France, 1964.

8. G. J. Minty, Monotone (nonlinear) operators in Hilbert space, Duke Math. J. 29 (1962), 341-346.

9. - On a "monotonicity" method for the solution of nonlinear equations in Banach spaces, Proc. Nat. Acad. Sci. U.S.A. 50 (1963), 1038-1041.

University of Chicago 